\title{
To Study Impact of Artificial Intelligence on HR Practices
}

\section{OPEN ACCESS}

Manuscript ID:

COM-2021-09044134

Volume: 9

Issue: 4

Month: October

Year: 2021

E-ISSN: 2582-6190

Received: 26.07.2021

Accepted: 14.09.2021

Published: 01.10.2021

Citation:

Kiran Kumar, M., and R. Elangovan. "To Study

Impact of Artificial Intelligence on HR Practices." ComFin Research, vol. 9, no. 4, 2021, pp. 34-38.

DOI:

https://doi.org/10.34293/ commerce.v9i4.4134

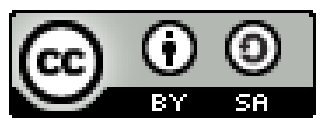

This work is licensed under a Creative Commons Attribution-ShareAlike 4.0 International License

\section{Kiran Kumar M}

Assistant Professor, Department of MBA, CIMS B-School

Jayanagar, Bengaluru, Karnataka, India \&

Research Scholar, Annamalai University, Tamil Nadu, India

D https://orcid.org/0000-0003-2084-9418

\section{R. Elangovan}

Professor \& Co-ordinator, Commerce Wing, Directorate of Distance Education Annamalai University, Annamalai Nagar, Tamil Nadu, India

\section{Abstract}

This studypresentsthe utilization of Artificial Intelligence in HR Practicesas a result of ever changing technology in the various business sector. Most of the businesses have implemented artificial intelligence to ascend the human resources efficiency in all sectors. The initiative starts from automated recruitment process till employee's performance appraisal. Organization leaders and HR executives have invested enormous faith incombining artificial intelligence (AI) withvarious functions of HRprocess like on-boarding, inductions, orientations, $T \& D$, compensation management shall and will progress the overall experience of an employee.Human Resource Management is drifting away from its fundamental administration functions like recruitment\& selection, Training \& Development, Appraisals to tremendouslysophisticated progress like Automations, Augmented Intelligence, Robotics and AI that are entirely redefining and reshaping the framework and characteristics of workforce and organizations. At, present the worldrevolving around AI and how it is absolutely manipulating the process of HR, and how AI is taking away lakhs of jobs in various sector globally. This article makes an attempt to discuss both the views that is in what way people sense,AIto be a boon and alsoriskfortheir jobs. We arepaying attention on the potential benefits and challenges of AI in the current scenario in industries. The study provides a brief knowledge of the future aim of artificial intelligence.

Keywords: Artificial intelligence, HR practice, Automation, Recruitment and selection, Efficiency

\section{Introduction}

Artificial intelligence is a computer term which is broadly used for software, computers and machines. During 1920, a science based fictional play named Rossumovi Univerzální Roboti which means- Rossum's Universal Robots, also better known as R.U.R. by Czechoslovakianauthor Karel Capek the term ROBOT was firstly coined. The term artificial intelligence was firstly introduced by 'John McCarthy' in 1956 in his first academic conference on the subject. But this journey of knowing this area in more depth had started much before than that.

Artificial intelligence is a method that utilizes human intelligence in multiple sectors and a budding technology that is used in all industries to increase productivity and progress performance. With utmost power AI tends to perform like brain of a human and also provides magnificent efficiency. Even though the inputs are different it gives specific outputs in HRM processes. Robotics are the machines that have capability to perform tasks like humans with AI that majorly deal with all the aspects of industry. 
So in simple terms we can say $\mathrm{AI}$ is the human intelligence performed by the machines where Intelligence refers to works in NLP, speech recognition, problem solving Decision analysis, etc.

Today's AI spreading rigorously. Now a days all business are entirely dependent on technologies. Much of the instruction for the activities to be performed by the human being are passed by intelligence system. Internet of things have changed our lifestyles to a greater extent. In present scenario mankind is majorly relying on technology even to perform the smallest of activity and this has resulted in escalation of the technological developments.

Personnel management is the way of engaging and monitoring people in the organization and it is about handling people and their behavior \&attitudes along with the subordinates and superiors in the organization, it is majorly connected with process of recruitment \& selection, training \&development, compensation, fringe benefits \& insurance etc.,

Human Resource Management is vital for the organization as it deals with all the process related to a personnel from hiring to firing, entry to exit of the same personnel. A healthy HRM encourages workers to excel their work for the organization and it will be able to achieve productivity in turn company's vision $\&$ mission, goals and objectives, quality policies. HRM plays a vital role in between managing the conducive atmosphere in the organization in properly channelizing all the information of the organizations. This communication established between the employee and employer is crucial and done with HRM.

In today's generation Artificial intelligence plays major role in process of HRM by reducing the workload of HR manager because AI uses chat bots algorithms and agnostic solution and based on algorithms for the various process, the process like recruitment \& solution, training \& development, compensation \& rewards takes place. Processes of HRM can be enhanced by associating AI to these activities and in turn brings a sort of enthusiasm in work for the employees.

AI helps HR manager by minimizing the bulk of manual efforts and paper work so as to perform tasks easily. AI helps in assisting the leave and absentees management of the employees. AI cloud helps the HRM easy by making sure the details of each employee is made available at any point of time to all with an access permission. AI have its own importance in HRM because of its unimaginable performance and results henceforth AI is turning to be the lifeline in most of the organizations that could help in minimizing the load on the employees. AI helps in screening the profiles of the candidates for the positions the organization looking for and it helps in reducing the work load of the recruiter. Even though AI is a budding technology it has higher influence in most of the processes as it helps in reducing the difficulties and job much easier.

\section{Meaning and Definition}

According to the father of Artificial Intelligence, John McCarthy, it is "The science and engineering of making intelligent machines, especially intelligent computer programs".

Definition: Artificial Intelligence is a way of making a computer, a computer-controlled robot, or a software think intelligently, in the similar manner the intelligent humans think.

$\mathrm{AI}$ is accomplished creating a training set to the machines for issues or the concepts on the basis of the human analysis and decisions to make decision on its own. After the machines are trained with the training set, the machines and software will be ready to apply those human intelligence on the test sets.

\section{Need of the Study}

The current scenario in industries is entirely changing where it is technology dependent and also the automations. Now a days all the dimensions strength performance and effectiveness is affected by the implementation of AI like superior technology across the globe. AI technology as proved itself to be fruition in various sectors like production, operation, marketing, financing, banking, insurance, administration, corporate by providing the higher productivity and efficiency. Thus this research work studies on how AI influences the practices of Human Resources in various sectors.

\section{Research Methodology}

This paper is purely based on conceptual study. Based on the secondary data and the research articles, that are published on Artificial intelligence and HR policies. No statistical analysis is used. 


\section{Statement of the Problem}

Today majority of the organization depend on the automation and machine learning, in present situation organizations are looking for best quality services for their buyer due to competition. Technology has going to create a tremendous changes in the many activates in many industries and organizations, so organizations are adopting technology but they are facing lot of issues and challenges. There are almost all the sectors like, IT, Banking, Hotel, Hospital, Teaching, and so on. This article is not restricted to one sector because HR Practices are similar from company to company/ organization to organization/ industry to industry.

\section{Objectives}

- To assess the influences of Artificial Intelligence on HR Practices.

- To analyze the challenges and limitations of Artificial Intelligence in HR Practice.

- To provide suggestions to increase the competence in HR Practice.

\section{Review of Literature}

Anupam Jauhari: 2017- AI adaptation and its Impact on HR practices: This paper summarizes how machine learning (ML) and artificial intelligence (AI) is impacting the HR practices day by day. AI has become a very important aspect in influencing how the various companies are hiring candidates and how they carry out each of their activities. Recruitment has become easy even for the people with no experience because of the way the ML technology makes use of the chat bots to solve the existing problems and proceed with the next activity. With the help of AI, the resumes of the candidates are screened and a selected mail or a rejection mail is sent automatically to all the candidates. Since the HR managers in the organizations are held up with a lot of other works, they must be having the knowledge of using AI and ML chat bots to get a solution for their problem. One of the major challenges that the HR's of all the organizations face is that of performance appraisal. In this process the employees are rated for their performance for the entire year and are given an appraisal which decides the career path of an employee.

Peter: 2018- Impact of AI on HR: This paper helps us in understanding how the implementation of AI in the companies will help them in bringing radical changes while executing the HR practices. The fact is that with the use of AI and ML there are many people who will be losing their jobs, but with the use of these technologies the organization becomes a high-yielding organization. Many of the Western organizations are positive in amalgamating AI with HR practices and have proved that they have become more productive. Even after seeing a lot of western organizations using $\mathrm{AI}$ and $\mathrm{ML}$ and become productive, there are a lot of organizations who are worried about the investment aspects. Recruitment becomes the best HR practice in integrating AI, because AI helps in various steps of recruitment which includes sourcing of the resumes and screening of those resumes. And then it is the responsibility of the Government and the organizations to influence, up-skill and reform their personnel to be more competent when compared to these technologies.

Dr. Surbhi Jain: 2018- HRM and AI: With this paper one can understand that HR in the past used to concentrate on the supervision of its staff in the organization, now gradually it has moved its concentration to fabricate a company that adjusts itself to the possible changing conditions, since change in condition will have an impact on the company. The AI is rejuvenating the company in this computerized world so the HR should rethink as per the needs that arise. AI is a down to earth presentation by machines in close relationship with insight of people. HR should educate the workers about the new advances all together for better future. Transparency has gotten standard in the realm of HR, workers can look at their pay rates against those of comparative employments utilizing a portion of the instruments like pay, organization scale and so forth. There are numerous difficulties in adjusting AI in an organization, HR specialists ought to likewise set themselves up so as to confront the dangers that emerge like joblessness, imbalance, racist robots, security, and so forth and also AI does not have a few highlights of good human judgment.

Dr. Jeremy Nunn: 2019- Emerging impact of AI on HR: This paper tells that at present AI has gotten significant in pretty much every industry. It has become the fantasy of each HR to utilize AI in their company because AI predominantly computerizes correspondence with the candidates. The two significant things in $\mathrm{AI}$ is that it fundamentally 
disposes the biasness while choosing the candidates and expands the effectiveness of the applicants. AI principally helps in screening process. It investigates the competitor's profiles and collaborates with them and takes a choice of choosing the correct candidate. It basically lessens the weight of work and the fundamental spotlight will be on the top competitors. AI can do facial acknowledgment and furthermore it directs the interviews basing upon training levels and their capacity and so forth.

Ruby Merlin. P, Yayam. R: 2018-AI in HRM: This paper emphasizes that AI will assist us with making our activity simple and diminish the outstanding burden of HR manager, however it doesn't replace the work which is crafted for a HR manager. ML has another wave in business. It fundamentally reveals to us that by 2020 each of the company will utilize AI consistently in light of the fact that HR divisions will confront new issues and difficulties, which implies the issues begins from choosing the correct candidate and retaining of that talent. HR experts will gradually rely upon the advancements like AI to climb up the ladder in their work. AI for the most part is utilized in recruitment process like it predominantly dismisses the resumes which are unfit. Artificial intelligence will utilize performance management like framework persistently taking input from the representatives. Employee retention is progressively significant in the company on the grounds that as indicated by the prerequisites of representatives the HR supervisor should actualize new strategies and techniques with the goal that workers will have enthusiasm to work in the company. Through ML we have numerous outcomes and certainly it has sway in this day and age.

\section{AI and its Impact on Various Occupations}

This paper summarizes that, AI helps the HR experts to accomplish their work in increasingly simple manner. This AI has emphatically affected a few angles like, improving the recruiting and direction process. Talent sourcing is one of the significant capacity of AI in HR. Artificial intelligence reinforce HR groups, they can check and procedure through an increasingly number of resumes to distinguish the best among them. Escalating the selection and induction procedures: Sourcing the talent is one amongst the majortask of $\mathrm{AI}$ in practices of $\mathrm{HR}$. AI fosters HR teams, they can verify and screen through alot number of profiles to fetch the best among them. With the help of AI the businesses will not only be able to save time but also choose the potential candidate for the opening position after assessing the fundamentals like skill sets, experience, background, remuneration etc. Every employee will have his own ways and preferences of learning the process, thus analyzing this behaviour of the employee personalization or customization in knowledge imparting of employees is established thus we can say it is a one of the major impact of AI. Retainingthe talent and enduring of employee satisfaction: employee turnover is the major problem for any organization thus retaining employees is major aspect and also the highly complicated one so AI assists HR personnel in focusing on employee performance, stress management, work life balance etc., and efforts are put to generate sense of belongingness towards the organization without bothering to the fringe benefits provided by the firm. Prediction: Anticipating the future turnover of employee is most essential. Determining the employee turnover is not an easy task to perform by the HR personnel thus AI come in handy in this situation by using the past data and anticipate the future of the employees move accurately, than ever before. This details gathered shall be useful to suggest and recommend to the organization for modification.

\section{Challenges and Limitations}

Even after successful implementation of Artificial Intelligence to HR practices there are multiple challenges to be faced. They vary from realistic to theoretical, as we know the data science, analytics and analyses concepts are still in its nascent stage of the growth the results of process that are provided by machine learning and Artificial Intelligence are not agile in nature and repeatability is not accurate, thus not completely reliable yet. Thus the decisions made with AI are not entirely dependable.

Companies are putting efforts to have highly returning Human Research strategies, especially for the high performing employees, so as to keep away from employee turning over rates. At times it 
is not easy to agree to and study various AI tools and techniques competently. This creates an obstacle in thriving the goals of organization. Human resources should be provided with the training and development at regular intervals on skills related to digital technology and AI. There are certain aspects of emotions and learning like phrases, signals, language, creativeness, empathy, team building of which it is very much significant for the firms to look for collaboration of AI with the HR practices so as you give better control and monitoring, thus creating conducive working environment in the current working scenario.

\section{Suggestions}

Today technology is broadly accepted by many of us, but still we are finding lot of issues in adopting AI in HR practices because we are not totallyaware about AI or machine learning. There is a solution to overcome from this problems. The organizations need to train their employees in the particular domain. Create awareness about AI and tell them it is not going to replace the human resource instead of that it make their job ease. Providing sufficient knowledge and making them to adopt HR applications and tools. It create an overall impact on the complete production system and it leads to success in their organization and improves the employee's efficiency.

\section{Conclusion}

AI based HR Practices have enormous potentiality to elevate productivity of employees and assisted HR personnel turned up knowledgeable consultants boosting performance of employee. AI fostered HR practices are resourceful enough and capable of analyzing, diagnosing, predicting, and also prescribing the solutions to the HR issues.

Any business or sectors should carefully analyze the procedures and processes of their organization before planning to incorporating the AI into the system as it could draw an adverse effect on the employees as it could affect the morale and functioning of the employees. Thus the businesses should be carefully looking after the issues arising from AI application to business.

\section{References}

Jauhari, Anupam. "How AI and Machine Learning will Impact HR Practices." VC Circle, 2017.

Reilly, Peter. The Impact of Artificial Intelligence on the HR function. Institute of Employment Studies, 2018.

Jain, Surabhi. Human Resource Management and Artificial Intelligence. 2018.

Nunn, Jeremy. "The Emerging Impact of AI on HR." Forbes, 2019.

Ruby Merlin, P., and R. Jayam. “Artificial Intelligence in Human Resource Management." International Journal of Pure and Applied Mathematics, vol. 119, no. 17, 2018.

Matsa, Prasanna, and Kusuma Gullamajji. “To Study Impact of Artificial Intelligence on Human Resource Management." International Research Journal of Engineering and Technology, vol. 6, no. 8, 2019.

Premnath, Eric, and Arun Antony Chully. "Artificial Intelligence in Human Resource Management: A Qualitative Study in the Indian Context." Journal of Xi'an University of Architecture \& Technology, vol. 11, no. 12, 2019.

Tewari, Isha, and Mohit Pant. “Artificial Intelligence Reshaping Human Resource Management : A Review." IEEE International Conference on Advent Trends in Multidisciplinary Research and Innovation, 2020.

Tiwari, Shivani. "Artificial Intelligence and Its Role in Human Resource Management." International Journal of Mechanical and Production Engineering Research and Development, vol. 10, no. 3, 2020.

Ahmed, Owais. "Artificial Intelligence in HR." International Journal of Research and Analytical Reviews, vol. 5, no. 4, 2018, pp. 971-978.

\section{Author Details}

Kiran Kumar M, Assistant Professor, Department of MBA, CIMS B-School, Jayanagar, Bengaluru, Karnataka, India \& Research Scholar, Annamalai University, Tamil Nadu, India, Email ID: kiranm@gmail.com

Dr. R. Elangovan, Professor \& Co-ordinator, Commerce Wing, Directorate of Distance Education, Annamalai University, Annamalai Nagar, Tamil Nadu, India 